\title{
Differential inhibitory effects of CrmA, P35, IAP and three mammalian IAP homologues on apoptosis in NIH3T3 cells following various death stimuli
}

\author{
Loretta Dorstyn $^{1}$ and Sharad Kumar ${ }^{1,2}$ \\ ${ }^{1}$ Hanson Centre for Cancer Research, Institute of Medical and Veterinary \\ Science, PO Box 14, Rundle Mall, Adelaide, SA 5000, Australia \\ 2 corresponding author: Sharad Kumar. tel: +61-8-8222 3738; fax: +61-8-8222 \\ 3139/+61-8-8222 3162; e-mail: skumar@immuno.imvs.sa.gov.au
}

Received: 18.3.97; revised: 19.5.97; accepted: 26.5.97

Edited by M. Piacentini

\begin{abstract}
We have investigated the effects of expression of the viral proteins CrmA, P35 and IAP, and the three mammalian IAP homologues (MIHA, MIHB and MIHC), on the regulation of apoptosis induced by either the overexpression of caspases (ICE, CPP32 and Nedd2), by serum-deprivation, or by $\gamma$ irradiation in NIH3T3 fibroblasts. As previously shown, CrmA strongly inhibited ICE-induced apoptosis but was ineffective against Nedd2- or CPP32-mediated apoptosis. P35, IAP and MIHA protected cells from apoptosis induced by the three caspases to varying extents but MIHB and MIHC were largely ineffective. NIH3T3 cells expressing P35 and MIHA, but not IAP, CrmA, MIHB and MIHC, showed enhanced cell survival under serum-deprived conditions. In addition, P35, CrmA and MIHA could provide substantial protection against death induced by $\gamma$-irradiation. These results suggest the presence of multiple apoptotic pathways with differential sensitivity to various naturally occurring apoptosis inhibitors.
\end{abstract}

Keywords: ICE; Nedd2; CPP32; caspase; P35; IAP

Abbreviations: ICE, interleukin- $1 \beta$-converting enzyme; PCR, polymerase chain reaction; $\mathrm{CrmA}$, cytokine response modifier A; TNF, tumour necrosis factor; TNFR, TNF receptor

\section{Introduction}

Apoptosis or programmed cell death (PCD) is a naturally occurring process of active cellular self-destruction that serves an essential function during development and in tissue homeostasis within multicellular organisms (Kerr et al, 1972). In addition, apoptosis also plays a role in eliminating virally-infected cells (reviewed in Vaux and Strasser, 1996). Although virus infection can itself trigger host cell apoptosis, many viruses encode anti-apoptotic proteins which function to prevent the death of the infected cell allowing further virus replication and thus facilitating persistent infection (reviewed in Shen and Shenk, 1995; Clem et al, 1996).

Three viral apoptosis inhibitory proteins, CrmA, P35 and IAP have been a subject of much interest recently. Cowpox virus encoded $38 \mathrm{kDa}$ CrmA (cytokine response modifier) protein shares homology with proteins of the serpin superfamily (Ray et al, 1992). CrmA directly inhibits some member of the ICE/CED-3 family of proteases (caspases) which are essential components of the cell death machinery (reviewed in Kumar, 1995; Martin and Green, 1995; Kumar and Lavin, 1996; Alnemri et al, 1996). Microinjection of CrmA into chicken neuronal cells affords partial protection from apoptosis induced by growth factor withdrawal (Gagliardini et al, 1994). In addition, CrmA is able to abrogate apoptosis induced by the activation of both TNF receptor and Fas (Tewari et al, 1995a). CrmA has also been shown to inhibit granzyme B and implicated in CTLmediated apoptosis (Quan et al, 1995; Tewari et al, 1995b).

Baculovirus P35 inhibits apoptosis in phylogenetically diverse organisms in response to a wide variety of apoptotic stimuli (reviewed in Clem et al, 1996). The expression of P35 is able to prevent developmentally programmed cell death in Drosophila melanogaster embryo as well as apoptosis in the eye of $D$. melanogaster upon X-irradiation (Hay et al, 1994). Expression of P35 in Caenorhabditis elegans prevents programmed cell death in developing larvae (Sugimoto et al, 1994) and microinjection of p35-expression vectors into rat sympathetic neurones can block apoptosis induced by the withdrawal of nerve growth factor (Martinou et al, 1995; Rabizadeh et al, 1993). Overexpression of P35 is also able to inhibit TNF- and Fasmediated apoptosis (Beidler et al, 1995). The mechanism of inhibition by P35 appears to lie in its ability to inhibit the proteolytic activity of various caspases (Bump et al, 1995; Xue and Horvitz, 1995).

A second baculovirus gene encoding a ring finger motif termed inhibitor of apoptosis (iap) was initially characterised in Cydia pomonella granulosis virus as being able to complement a p35 mutation (Crook et al, 1993). Homologues of IAP have been found in three baculoviral strains; Cydia pomonella granulosis virus (Cp-iap), Orgyia pseudotsugata (Op-iap) and Autographa californica (Ac-iap) nuclear polyhedrosis viruses (reviewed in Clem et al, 1996). Of the three $A c$-iap is the only gene unable to prevent virus-induced apoptosis in SF-21 cells in the absence of $p 35$ (Clem and Miller, 1994). All three proteins contain two conserved motifs, a carboxyl-terminal ring finger motif and a novel pair of 65 amino acid repeats termed BIRs (baculovirus IAP repeats) at the amino terminus (Birnbaum et al, 1994). Overexpression of OpIAP in BHK and N18 cells is able to inhibit apoptosis induced by Sindbis virus infection (Duckett et al, 1996). A 
Drosophila homologue of IAP (DIAP) has also been shown to inhibit normally occurring cell death in the Drosophila eye (Hay et al, 1995). Four mammalian homologues of IAP have been recently cloned. Neuronal apoptotic inhibitory protein (NAIP) was initially characterised on the basis of its association with spinal muscular atrophy (Roy et al, 1995) and shown to inhibit apoptosis by a number of stimuli (Liston et al, 1996). Three other mammalian IAP homologues MIHA/XIAP/hILP, MIHB/hIAP-1/C-IAP1 and MIHC/ hIAP-2/c-IAP2 have been described recently (Rothe et al, 1995; Listen et al, 1996; Uren et al, 1996; Duckett et al, 1996). These proteins contain the conserved three $\mathrm{N}$ terminal BIR repeats and a single C-terminal RING finger domain. The precise function of the IAP homologues is obscure but MIHB/C-IAP1 and MIHC/C-IAP2 have been shown to interact with TNF receptor-2 (TNFR2) associated factors 1 and 2 (TRAF1/TRAF2) (Rothe et al, 1995; Uren et al, 1996). A recent study suggests that TRAF2 and c-IAP1 are a part of the TNFR1 signalling complex (Shu et al, 1997). The recruitment of both proteins is mediated by the death domain protein TRADD in a TNF-dependent manner (Shu et al, 1996).

Despite various published reports on the effect of various viral and mammalian proteins on apoptosis, there has been no systematic study to compare the abilities of these proteins to inhibit apoptosis induced by various agents in a single cell system. In the present study, we have used transiently and stably transfected NIH3T3 cells to monitor the effects of the expression of $\mathrm{CrmA}, \mathrm{P} 35, \mathrm{Bcl}-$ 2, IAP, MIHA, MIHB and MIHC on apoptosis induced by either the overexpression of one of the three caspases (ICE, Nedd2 and CPP32), by serum deprivation, or by $\gamma$ irradiation. Varying degrees of inhibition by different proteins on apoptosis induced by a number of stimuli seen in this study suggests that various pathways of apoptosis in a single cell type utilise components (including different caspases) with differential sensitivity to inhibition by the caspase inhibitors (P35 and CrmA) and IAP-like proteins.

\section{Results}

\section{Differential effects of various inhibitors on apoptosis induced by the overexpression of three caspases}

To address the extent by which CrmA, P35, IAP and the three mammalian homologues of IAP are able to inhibit apoptosis induced by the overexpression of caspases, we chose three caspases ICE (caspase-1), Nedd2 (caspase-2) and CPP32 (caspase-3) for this study. These three caspases belong to three different subfamilies (Alnemri et al, 1996) and have been shown to induce apoptosis when overexpressed in mammalian and insect cells (Miura et al, 1993; Kumar et al, 1994; Fernandes-Alnemri et al, 1994). Although the mechanism by which caspase overexpression induces apoptosis is not entirely clear, it is believed that overexpression results in caspase activation by autocatalysis. Using Semliki Forest virus vector system Allet et al (1996) recently demonstrated that wild-type Nedd2 when overexpressed in $\mathrm{CHO}$ cells is cleaved into subunits, while a catalytically inactive mutant of Nedd2 was not processed. This suggested that activation of Nedd2 by autocatalysis is required for its apoptotic activity. We carried out immunoblot analysis using specific antibodies to check whether overexpression of caspases in our transient transfection assays also results in caspase processing. Although only a fraction of the cells are transfected in these assays and the results are somewhat complicated due to some cell death occurring due to lipofection (Kumar et al, 1994), we were able to see disappearance of the caspase precursors and/or appearance of subunits representative of active caspases in transiently transfected NIH3T3 and N18 cells (data not shown). As shown by Allet et al (1996), the catalytically inactive Nedd2 mutant which is unable to induce apoptosis (Kumar et al, 1994) was not processed in transfected cells (data not shown).

DNA fragments encoding various inhibitors and caspases were cloned into pCXN2, a mammalian expression vector carrying a chicken $\beta$-actin promoter (Niwa et al, 1991). As previously shown for Rat-1 cells (Miura et al, 1993) transient transfection of NIH3T3 cells with ICE induced apoptosis in the majority of the cell population (Figure 1A). Consistent with the recently published reports (Uren et al, 1996; Hawkins et al, 1996) co-transfection of CrmA, p35, IAP, MIHA and Bcl-2 resulted in potent inhibition of cell death induced by the transfection of ICE (Figure 1A). MIHB and MIHC also inhibited ICE-induced apoptosis, but the effects were much less pronounced. The effects of various inhibitors on Nedd2-induced apoptosis were very different from ICE-induced apoptosis (Figure 1B). As previously reported (Kumar et al, 1994), Bcl-2 partially protected cells from Nedd2-induced apoptosis, offering approximately $45 \%$ protection. P35 also provided significant protection ( $\sim 50 \%)$ from Nedd2 induced apoptosis, whereas partial inhibition $(20-25 \%)$ was evident with IAP, $\mathrm{MIHB}$ and MIHC (Figure 1B). Both CrmA and MIHA were unable to significantly inhibit Nedd2-induced apoptosis. Unlike ICE and Nedd2, overexpression of CPP32 was not effective in killing NIH3T3 cells, causing only $\sim 30 \%$ cell death. P35, IAP and MIHA inhibited this cell death by approximately $45 \%$ (Figure $1 \mathrm{C}$ ). On the other hand, Bcl-2 showed only $25 \%$ inhibition and CrmA, MIHB and MIHC had no significant inhibitory effect (Figure 1C).

\section{NIH3T3 cells stably transfected with viral and mammalian inhibitors}

NIH3T3 cell lines stably expressing CrmA, P35, IAP, MIHA, MIHB and MIHC were generated in order to determine whether long term expression of these proteins have any effect on apoptosis induced by various stimuli. CrmA, P35 and IAP expression were assessed by Northern blot analysis of total RNA from pooled populations of stably transfected NIH3T3 cells (Figure 2). In all cases, expected size mRNA for the transfected clones were clearly visible. Bcl-2 expressing NIH3T3 cell lines have been previously described (Kumar et al, 1994). MIHA, MIHB and MIHC protein expression was assessed by Western blotting using a monoclonal anti-FLAG antibody specific for the FLAG epitope that was incorporated by PCR in the cDNA (Figure 3). MIHA was detected as a 


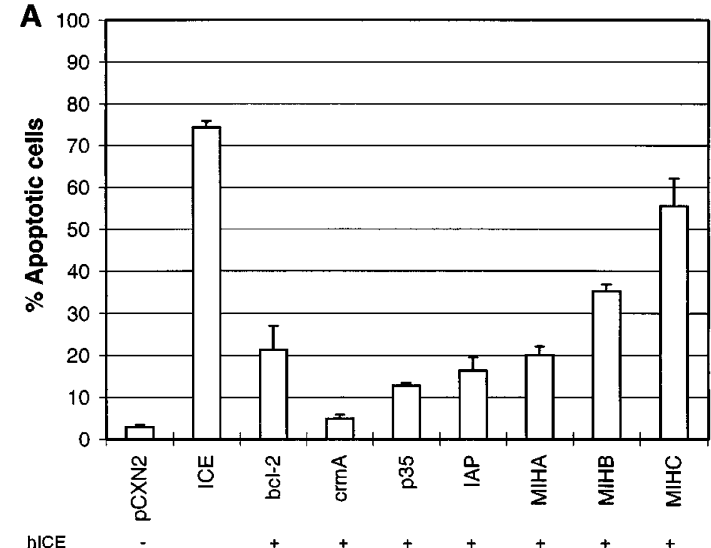

Expression constructs

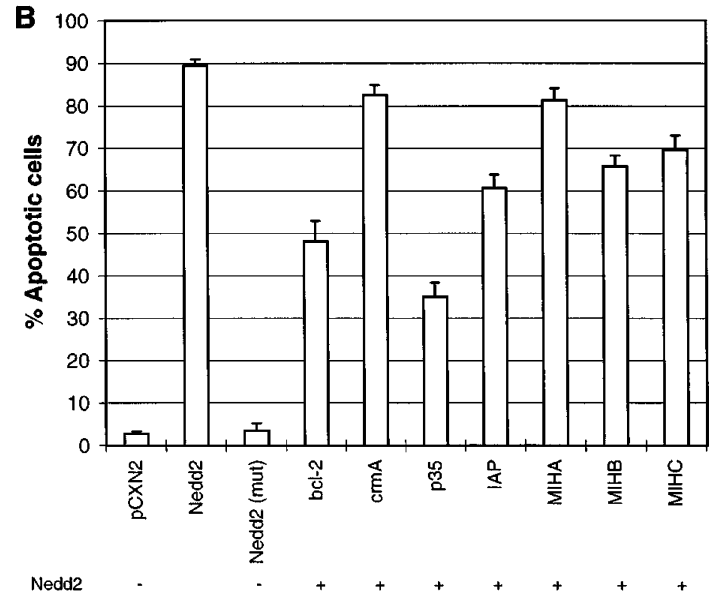

Expression constructs

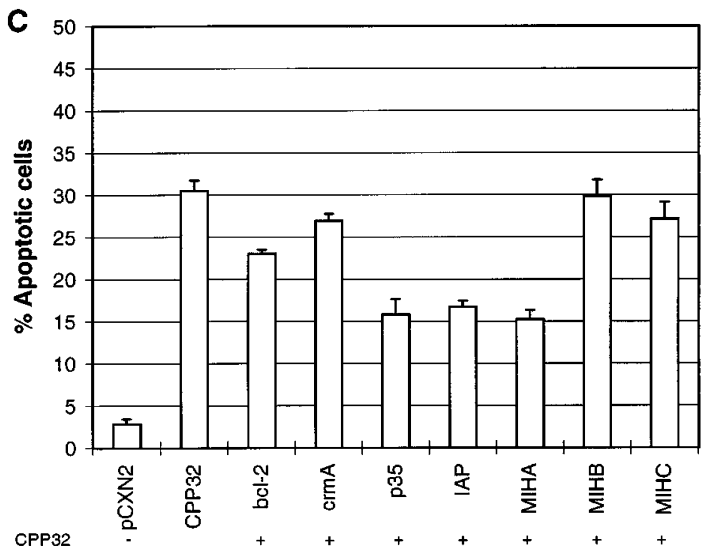

Expression constructs

Figure 1 The effect of various viral and mammalian proteins on apoptosis in NIH3T3 cells induced by the overexpression of ICE (A), Nedd2 (B) and CPP32 (C). Cells were transfected with the various viral and mammalian constructs as indicated. Cotransfection with either ICE, Nedd2 or CPP32 is indicated (+). On average $>400$ blue cells were counted for each construct co-transfected with pCXN2-caspase construct. The data are represented as means \pm S.E.M. taken from three independent experiments. Nedd2(mut) encodes a nonfunctional Nedd2 where the catalytic cysteine residue is altered to a glycine residue
$58 \mathrm{kDa}$ protein and as can be seen both in pooled population of transfectants and clones derived from the transfected cells (Figure $3 \mathrm{~A}$ ). MIHB and MIHC both ran as approximately $68 \mathrm{kDa}$ proteins and once again, the majority of clones expressed the respective proteins (Figure 3B, C). In the following experiments we have used both the pooled transfected cells and two selected clones expressing relatively high and relatively low levels, respectively, of MIHA, MIHB and MIHC proteins.

\section{Caspase induced cell death in NIH3T3 cells expressing various inhibitors}

NIH3T3 cell stably expressing CrmA, P35, Bcl-2, IAP, MIHA, $\mathrm{MIHB}$ and $\mathrm{MIHC}$ were transiently transfected with ICE, Nedd2 or CPP32 and assessed for the extent of inhibition of apoptosis mediated by the various viral and mammalian apoptosis inhibitors. With the exception of CrmA, in each case, the percentage inhibition of caspase-mediated apoptosis by each viral and mammalian protein studied, appears to be on average $10-15 \%$ less than demonstrated for transient co-transfection assays, and this may be due to the relatively lower levels of expression of the proteins in the stably transfected NIH3T3 cells (compare Figures 1 and 4). As evident in Figure 4, the levels of inhibition of ICE-induced apoptosis by MIHA, MIHB, MIHC, IAP and P35 were similar to those observed in transient co-transfection assays (Figure 1A). There did not appear to be any differences in the level of inhibition of Nedd2-induced apoptosis by any of the proteins assessed with the exception of CrmA and MIHA which showed a slightly higher level of protection compared with the transient co-transfection experiments (compare Figures 1B and 4B). Apart from a slightly enhanced protective effect by
A

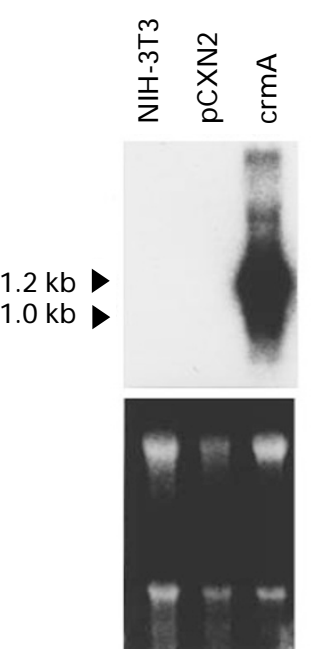

B

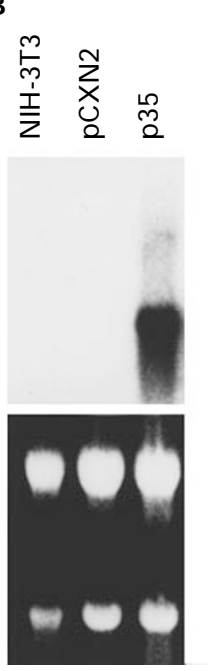

C

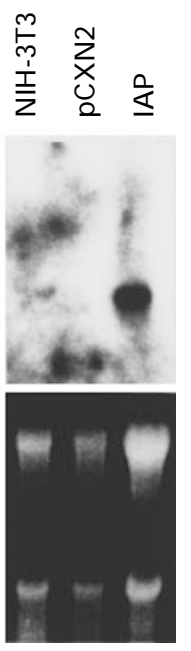

Figure 2 mRNA expression analysis of NIH3T3 cells stably transfected with CrmA (A), p35 (B) and Op-iap (C). In the upper panel total cellular RNA samples from untransfected cells (NIH3T3) and pooled transfectants were analyzed by Northern blotting. The lower panel shows the ethidium bromide stained gels prior to blotting. Lanes marked pCXN2 contained RNA isolated from vector transfected NIH3T3 cells. As indicated, the sizes of CrmA, p35 and IAP transcripts are approximately $1.2,1.0$ and $1.0 \mathrm{~kb}$, respectively 


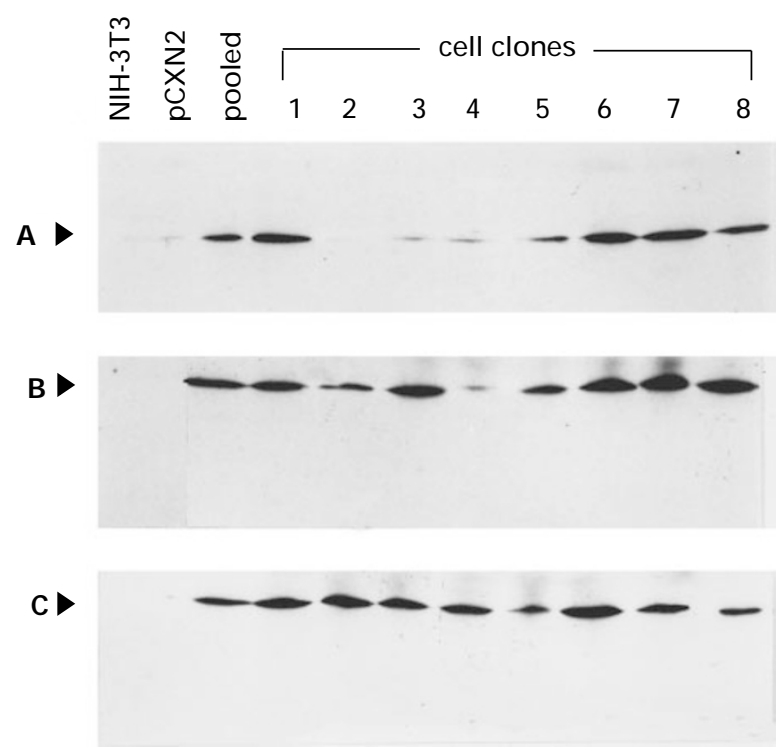

Figure 3 Expression analysis of $\operatorname{MIHA}(\mathbf{A}), \operatorname{MIHB}(\mathbf{B})$ and $\mathrm{MIHC}(\mathbf{C})$ in transfected NIH3T3 cells. Protein expression was assessed by Western blotting with the anti-FLAG monoclonal antibody M2. Results are displayed for pooled populations of NIH cell stably transfected with each $\mathrm{MIH}$ construct as well as clones of each transfected cell type. Total protein was used for all Western analyses. NIH3T3 denotes protein from non-transfected NIH3T3 cells; pCXN2 denotes protein extracted from pCXN2 vector transfected NIH3T3 cells; pooled denotes protein extracted from pooled populations of transfected cells; numbers 1-8 denote selected clones of transfected cells. The approximate sizes of MIHA, MIHB and MIHC are 58,68 and $68 \mathrm{kDa}$, respectively

Bcl-2, there was no significant variations in the inhibitory activity of CPP32-induced apoptosis by the overexpression of any of the viral or mammalian proteins (Figure 4C). Lastly, clones expressing different levels of MIHA, MIHB and MIHC did not show any significant differences in the level of protection against caspase-induced apoptosis (Figure 4).

\section{Inhibition of apoptosis induced by serum deprivation}

NIH3T3 cells stably transfected with various expression constructs were subjected to serum deprivation over a five day period and subsequently assessed for the extent of survival. As expected, $\mathrm{Bcl}-2$ expressing cells were particularly resistant to serum-depletion induced apoptosis (Figure 5A). P35 expression also conferred substantial resistance to cell death induced by serum-withdrawal, as evident by a delayed onset of apoptosis, but CrmA and IAP offered no observable protection (Figure 5A). NIH3T3 cells expressing MIHA showed a delay in the onset of apoptosis under serum-free conditions and significant differences in cell survival (20$30 \%$ ), as compared to control cells, were evident in the first 2 days following serum withdrawal (Figure 5B). However, by day 4, majority of both the control and the MIHA expressing cells had undergone apoptosis and no significant differences in cell viability were obvious (Figure $5 \mathrm{~B}$ ). MIHB and MIHC did not offer substantial protection of cells from death induced by the absence of serum (Figures $5 \mathrm{C}$ and D). CrmA has been previously shown to inhibit serum- and NGF-deprivation induced apoptosis in Rat-1 and neuronal cells, respectively
(Wang et al, 1994; Gagliardini et al, 1994). In contrast to these findings, in our experiments, expression of CrmA in NIH3T3 cells failed to significantly protect these cells from apoptosis induced by serum-deprivation (Figure 5A). To check whether this may be a cell type specific effect, we transfected pCXN2CrmA into a neuroblastoma cell line $\mathrm{N} 18$, and subjected the CrmA expressing cells to serum withdrawal. In two separate N18 clones expressing levels of CrmA mRNA comparable to those in NIH3T3-CrmA transfectants (data not shown), substantial protection of apoptosis was clearly evident (Figure 6). These results, and the results shown below with $\gamma$-irradiation suggest that the lack of inhibition of serumdeprivation induced apoptosis by CrmA may be an intrinsic feature of NIH3T3 cells.

\section{$\gamma$-irradiation induced apoptosis in NIH3T3 cells expressing various inhibitory proteins}

In further experiments, NIH3T3 cells expressing P35, CrmA, IAP, Bcl-2, MIHA, MIHB and MIHC were analyzed for their resistance to apoptosis induced by $\gamma$-irradiation. Preliminary studies indicated that NIH3T3 cells treated with 30 Gy of $\gamma$ irradiation take at least 3 days to show significant levels of cell death, therefore the following experiments were performed over a five day time course. As expected, Bcl-2 conferred substantial resistance to $\gamma$-irradiation as demonstrated by the significantly higher level of cell survival compared to controls (Figure 7A). CrmA and P35 also provided significant protection $(20-40 \%)$ against $\gamma$-irradiation induced apoptosis whereas IAP was an ineffective death inhibitor (Figure 7A). NIH3T3 cells expressing MIHA were significantly more resistant to $\gamma$-irradiation induced apoptosis compared to untransfected cells (Figure 7B). NIH3T3 clones expressing varying levels of MIHA showed minor variations in their resistant to radiation-induced death, but there appeared to be no significant correlation between the level of protection and MIHA expression (Figure 7B). Pooled populations and clones of MIHB and MIHC transfected NIH3T3 cells expressing varying levels of proteins failed to show any resistance to irradiation-induced apoptosis as compared to untransfected NIH3T3 cells (Figure 7C and D).

\section{Discussion}

The results of this study can be summarised as follows: (1) Viral proteins P35 and CrmA inhibit different members of the caspase family to varying extents. While CrmA is a potent inhibitor of ICE-induced apoptosis, it is relatively ineffective against CPP32 and Nedd2. P35 can inhibit several caspases, albeit with low efficiency. (2) IAP inhibited apoptosis induced by the three caspases used in this study to a certain extent, but overall, the inhibition was weak as compared to ICE inhibition by CrmA. (3) The three mammalian homologues of IAP showed differing abilities to inhibit apoptosis induced by the three caspases. While MIHA inhibited ICE and CPP32 induced apoptosis, it was ineffective against apoptosis induced by the ectopic expression of Nedd2. MIHB and $\mathrm{MIHC}$ were much less effective against all three caspases, although both showed partial inhibition of ICE-induced apoptosis. (4) Except for Bcl-2 and P35, and to some extent 

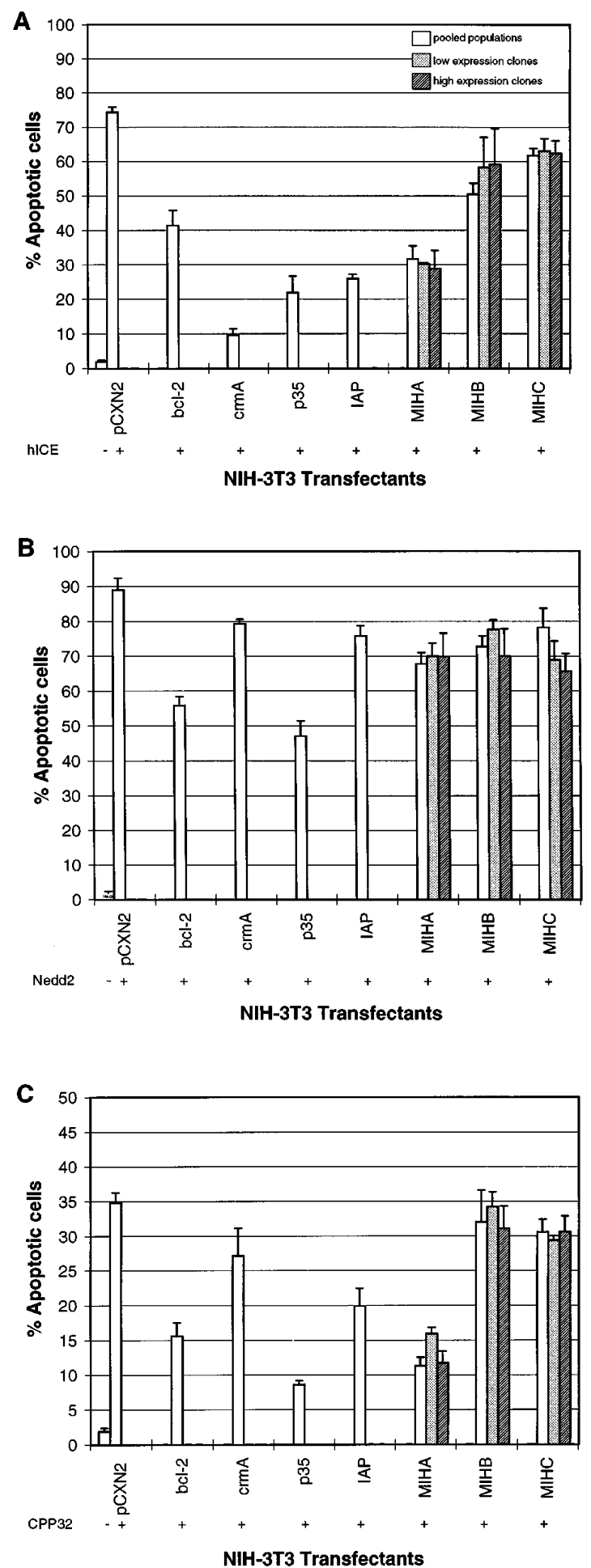

Figure 4 Induction of apoptosis by ICE (A), Nedd2 (B) and CPP32 (C) in NIH3T3 cells stably expressing various viral or mammalian proteins. Data (means \pm S.E.M.) were derived from three independent experiments. Cotransfection with each caspase is indicated $(+)$. On average $>400 \beta$ galactosidase positive blue cells were counted in each case. Shaded and striped columns represent clones expressing relatively low and high levels, respectively, of MIHA, MIHB and MIHC proteins
MIHA, all other proteins were unable to inhibit or delay apoptosis of NIH3T3 cells under serum-deprived conditions. While CrmA efficiently inhibited the apoptosis of a neuroblastoma cell line, it was unable to do so in NIH3T3 cells. (5) Finally, CrmA, P35, Bcl-2 and MIHA were able to delay the onset of $\gamma$-irradiation induced apoptosis of NIH3T3 cells, while IAP, MIHB and MIHC were ineffective in doing so. These results, further summarised in Table 1, suggest that in NIH3T3 cells, multiple apoptotic pathways utilise different components of the apoptotic machinery, which in turn have differential susceptibility to inhibition by various naturally occurring proteins used in the present study.

Expression of the full length cDNAs for several caspases has been shown to induce apoptosis in cultured cell lines (reviewed in Kumar and Lavin, 1996). This is surprising since caspases require processing of the precursor into subunits for activation, and normally growing cells contain caspase zymogens in modest concentrations. One possibility is that overexpression in transient transfection assays results in localised high concentrations of caspase precursor promoting autocatalysis. Whatever the mechanism, overexpression by transient transfection provides a convenient experimental tool to study the effects of various molecules on caspase mediated apoptosis. Among the molecules used in the present study, only CrmA and P35 are known to directly inhibit caspase family members. Our study further confirms that these two viral proteins have very different effects on different members of the caspase family. These differences are unlikely to be due to the different levels of expression of these proteins, as same expression vector and cell type was used in all cases. Furthermore, NIH3T3 cells stably expressing various inhibitors, when transiently transfected with ICE, Nedd2 or CPP32, show levels of apoptosis comparable to shown in experiments where caspase and inhibitor were transiently co-expressed, suggesting that the inhibitor concentration was not a limiting factor in these studies. Co-expression of $\mathrm{Bcl}-2$ provided significant protection against ICE and Nedd2 induced cell death. Bcl-2 has been shown to be upstream of the PARP cleaving caspase (eg. CPP32 or Mch3) and may lie downstream of caspases such as ICE and Nedd2. Unlike ICE and Nedd2, the apoptosis inducing activity of CPP32 was much weaker perhaps because CPP32 needs to be activated through the action of another upstream caspase.

In a previous study using transient transfection assay, IAP was shown to inhibit apoptosis induced by ICE and ICH-1 but not ICE-LAP3 (Hawkins et al, 1996). In cotransfection assays ICE-induced apoptosis was also shown to be inhibited by MIHA and MIHB, but not MIHC (Uren et al, 1996). Consistent with Hawkins et al, (1996) our results with either transiently transfected or stably transfected NIH3T3 cells indicate that IAP and MIHA inhibited apoptosis induced by the overexpression of ICE. On the other hand, all three IAP homologues were ineffective against Nedd2-induced apoptosis and only MIHA had any effect on CPP32-induced apoptosis. These results suggest that the modes of action of the baculovirus IAP and the three mammalian homologues are distinct from each other.

All three mammalian homologues of IAP have been shown to inhibit apoptosis of serum-deprived $\mathrm{CHO}$ cells (Liston et al, 

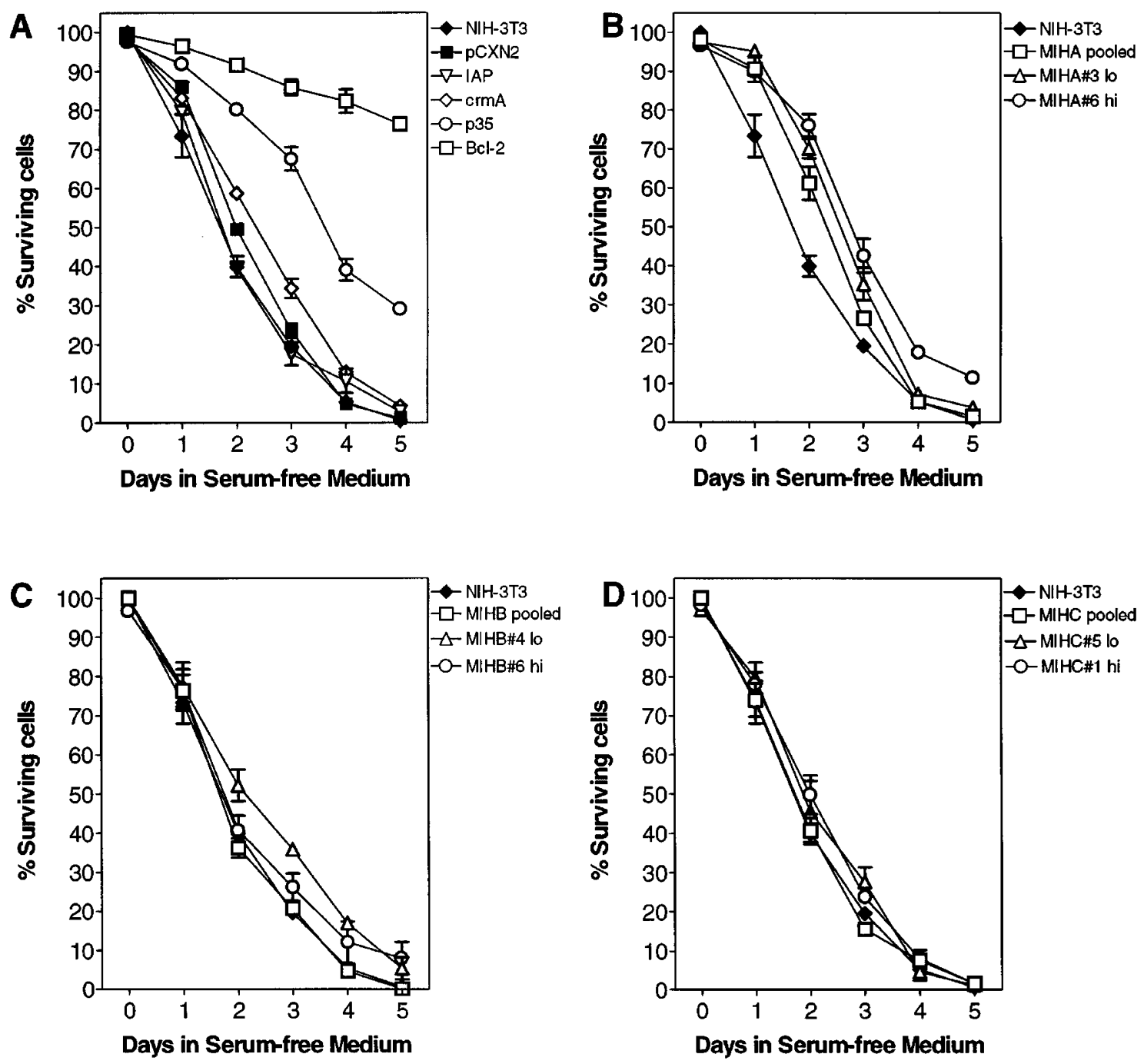

Figure 5 Serum deprivation induced apoptosis in NIH3T3 cell stably expressing Bcl-2, CrmA, P35, IAP, MIHA, MIHB and MIHC. Data (mean \pm S.E.M.), represent the percentage surviving cells as assesed by trypan blue exclusion. (A) The effect of CrmA, P35, IAP and Bcl-2 expression on the survival of cells following serumdepletion. (B, C and D) The effects of MIHA, MIHB and MIHC expression, respectively, on cell survival in serum-free medium. 'Pooled' denotes pooled populations of transfected cells; 'lo' denotes clones with low level of protein expression; 'hi' denotes clones with relatively high level of protein expression and clone numbers corresponding to those in Figure 3, are indicated. For Bcl-2, CrmA, P35 and IAP, only pooled population of transfected cells were used

1996). However, in stably transfected NIH3T3 cells, only MIHA showed some inhibitory effect, while IAP, MIHB and MIHC were completely ineffective. As expected from previous studies (reviewed in Cory, 1995), Bcl-2 expressing NIH3T3 cells were particularly resistant to serum-deprivation induced apoptosis. P35 also showed significant effects, but CrmA was unable to suppress apoptosis. Inability of CrmA to suppress serum-deprivation induced apoptosis of NIH3T3 cells is unlikely to be due to low expression of $\mathrm{CrmA}$, as these cells showed significant protection against $\gamma$-irradiation induced apoptosis. A neuroblastoma cell line expressing levels of CrmA comparable to those in NIH3T3/CrmA transfectants, showed substantial inhibition of apoptosis induced by serum withdrawal. Thus the lack of CrmA inhibition in NIH3T3 cells might suggest that these cells use a caspase not inhibited by CrmA in apoptosis under factor free conditions. Unlike for serum withdrawal, $\gamma$-irradiation-induced apoptosis in NIH3T3 cells could be significantly suppressed by CrmA and MIHA, in addition to $\mathrm{Bcl}-2$ and P35. In contrast, MIHB and MIHC provided little protection against death by ionising radiation consistent with their relative inability to inhibit apoptosis in transient transfection experiments.

While CrmA and P35 are known to inhibit apoptosis by directly inhibiting one or more members of the caspase family, nothing is known about the mechanism of action of IAP and its mammalian homologues. Two mammalian IAPlike proteins (MIHB/C-IAP1, and MIHC/C-IAP2) have been shown to interact with TRAF1 and TRAF2, two proteins found in complex with the cytoplasmic domain of TNFR2 (Rothe et al, 1995; Uren et al, 1996). However, both MIHB and $\mathrm{MIHC}$, which are highly related to each other, appear to be least effective in inhibiting apoptosis induced by various 


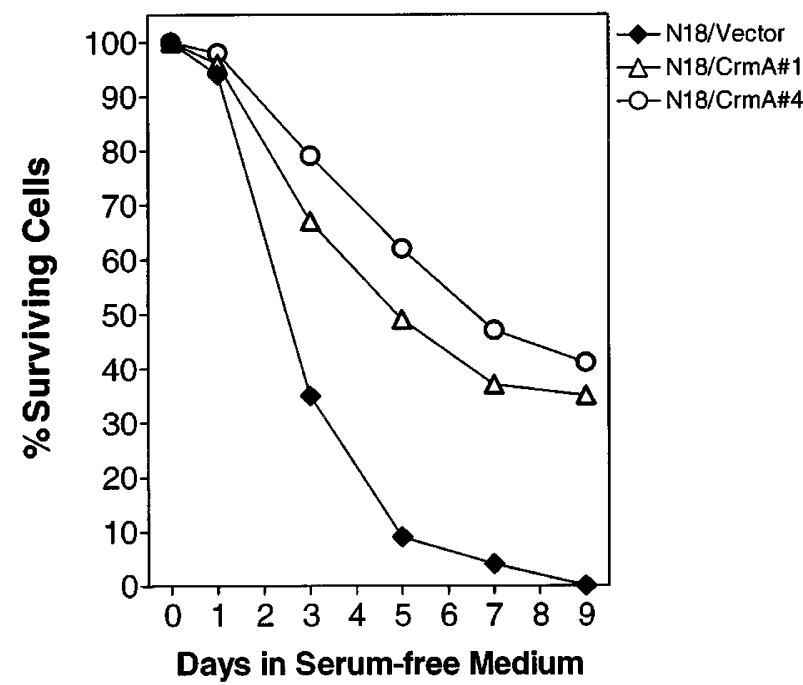

Figure 6 Survival of CrmA expressing N18 cells under serum-free conditions. Pooled vector ( $\mathrm{pCXN2}$ ) transfected N18 cells or two N18 clones (no. 1 and 4) transfected with pCXN2-CrmA were exposed to serum-free conditions for the indicated period of time and cell viability determined by trypan blue exclusion. Data are derived from an experiment performed in duplicate

death stimuli. Although IAP inhibited apoptosis induced by transient overexpression of caspases, it was ineffective in protecting NIH3T3 cells from serum-withdrawal and $\gamma$ irradiation induced apoptosis. Whether this effect is due to the lower expression or poor protein stability in transfected NIH3T3 cells remains to be seen. Nevertheless, these results suggest that despite their structural similarities, all IAP-like proteins are not necessarily involved in the regulation of apoptosis and some may function in unrelated pathways. This is also consistent with the observation that IAP homologue in the insect virus $A$. californica is unable to inhibit apoptosis (Clem and Miller, 1994).

\section{Materials and Methods}

\section{Construction of expression vectors}

For the reasons of uniformity, all expression plasmids were generated in the pCXN2 vector background (Niwa et al, 1991) by cloning the coding regions downstream of the chicken $\beta$-actin promoter. Plasmids containing full length coding region of Nedd2 (pCXN2-N2), mutated Nedd2 encoding a catalytically inactive protein (pCXN2-N2 Gly319) and Lac-Z (pEF- $\beta$ gal) have been described previously (Kumar et al, 1994). cDNAs for MIHA, MIHB, MIHC in pGEM7 vector and Op-IAP in $p E F p u r o$ were a generous gift of Dr David Vaux. Coding regions of MIHA, MIHB and MIHC were amplified by PCR using primers with terminal EcoRI sites and a $3^{\prime}$ FLAG-tag sequence and cloned into EcoRI site of pCXN2. Op-IAP was released as a $0.8 \mathrm{~kb} \mathrm{BamHI/Xbal}$ fragment and cloned blunt ended into $\mathrm{PCXN} 2$ after T4 polymerase treatment. DNA fragment encoding CrmA (a kind gift of Dr David Pickup) was isolated as a $1.1 \mathrm{~kb}$ EcoRI/Hindlll fragment from pGEM7 vector, treated with T4 polymerase and cloned blunt ended into pCXN2. P35 and CPP32 coding DNA were released from the pcDNA3 constructs (kindly provided by Dr VM Dixit) as EcoR1/Xbal fragments and cloned into PCXN2 after T4 polymerase mediated end flushing. Human ICE cDNA (kindly donated by Dr D Miller) was released from pGEM3 as a $1.2 \mathrm{~kb} E c o R I$ fragment and cloned into the EcoRI site of pCXN2.

\section{Transient transfection assays}

Cells were maintained and transfected as described previously (Kumar et al, 1994). For transfection, cells were plated in six well trays at a density of $2 \times 10^{5}$ cells/well. The following day, the expression vectors were co-transfected in a $1: 1$ ratio with either pCXN2-Nedd2, pCXN2-ICE or pCXN2-CPP32 expression plasmids and $0.5 \mu \mathrm{g}$ of pEF- $\beta$ gal plasmid to total $3 \mu \mathrm{g}$ DNA. Transfections were carried out using a cationic lipid reagent (DOSPER, Boehringer Mannheim). DNA was mixed in a 1:4 ratio with DOSPER using the following procedure: $12 \mu$ LOSPER per reaction, was mixed with HEPES-buffered saline (HBS) to a final volume of $50 \mu \mathrm{l}$ and incubated at room temperature for $20 \mathrm{~min}$. $3 \mu \mathrm{g}$ DNA expression constuct mixture (as above) was made up to $50 \mu \mathrm{l}$ with HBS. $50 \mu \mathrm{l}$ DOSPER mix was added to DNA, mixed gently and incubated at room temperature for $20 \mathrm{~min}$ for a lipid/DNA complex to form. Cell monolayers were washed in $1 \mathrm{ml}$ of OPTI-MEM medium (Life Technologies) and then fed with $1 \mathrm{ml}$ OPTI-MEM. The $100 \mu \mathrm{l}$ DOSPER/DNA complex was added drop wise to the cells, swirling the tray simultaneously to ensure an even distribution of the mixture, and the cells were incubated at $37^{\circ} \mathrm{C}$. Five hours later, $1 \mathrm{ml}$ OPTI-MEM containing $20 \%$ foetal calf serum (FCS) was added and cells incubated at $37^{\circ} \mathrm{C}$ overnight. $20 \mathrm{~h}$ post-transfection, cells were fixed $(2 \%$ formaldehyde, $0.2 \%$ glutaraldehyde in PBS) and stained with $0.1 \% \mathrm{X}$-gal, $5 \mathrm{mM}$ potassium ferricyanide, $5 \mathrm{mM}$ potassium ferrocyanide, $2 \mathrm{mM} \mathrm{MgCl}_{2}$. $\beta$-galactosidase positive blue cells were counted at random $4-8 \mathrm{~h}$ poststaining, and percentage apoptotic cells assessed by morphological examination.

\section{Stable transfections}

NIH3T3 cells were plated at a density of $5 \times 10^{5}$ cell in $60 \mathrm{~mm}$ dishes. The following day, $5 \mu \mathrm{g}$ expression vector encoding cDNAs for MIHA, MIHB, MIHC, OpIAP and CrmA were individually mixed in a 1:5 ratio with $10 \mu$ lipofectamine lipid reagent (GIBCO) and the volume made up to $150 \mu \mathrm{l}$ with sterile water. The mixture was incubated at room temperature for $20 \mathrm{~min}$ to allow the formation of DNA-lipid complexes. Cells were washed and refed in $3 \mathrm{ml}$ OPTI-MEM and the lipid-DNA complex added drop wise as above. After a $5 \mathrm{~h}$ incubation, $3 \mathrm{ml}$ OPTIMEM containing $20 \%$ FCS was added. At $24 \mathrm{~h}$ post-transfection, the medium was replaced with fresh DMEM $+10 \%$ FCS. After a further $24 \mathrm{~h}$ incubation, cells were split 1:10,1:20 and 1:50 and grown under selection for 1 week in DMEM + 10\% FCS and $1 \mathrm{mg} / \mathrm{ml} \mathrm{G418}$ (GIBCO-BRL). Where indicated, drug selected single colonies were picked and cloned. NIH3T3 cells expressing $\mathrm{Bcl}-2$ have been described elsewhere (Kumar et al, 1994). N18 cells were transfected with pCXN2 or pCXN2-CrmA under conditions similar to those described for NIH3T3 cells. After selection in G418 containing medium, single isolated colonies were picked and propagated.

\section{Cell death assays}

For serum withdrawal experiments NIH3T3 and N18 cells were plated at a density of $2 \times 10^{5}$ cells/well in a 6 -well tray. Semiconfluent cell monolayers were washed three times in PBS and refed with DMEM without foetal calf serum. At 24 or $48 \mathrm{~h}$ intervals, cells were harvested 

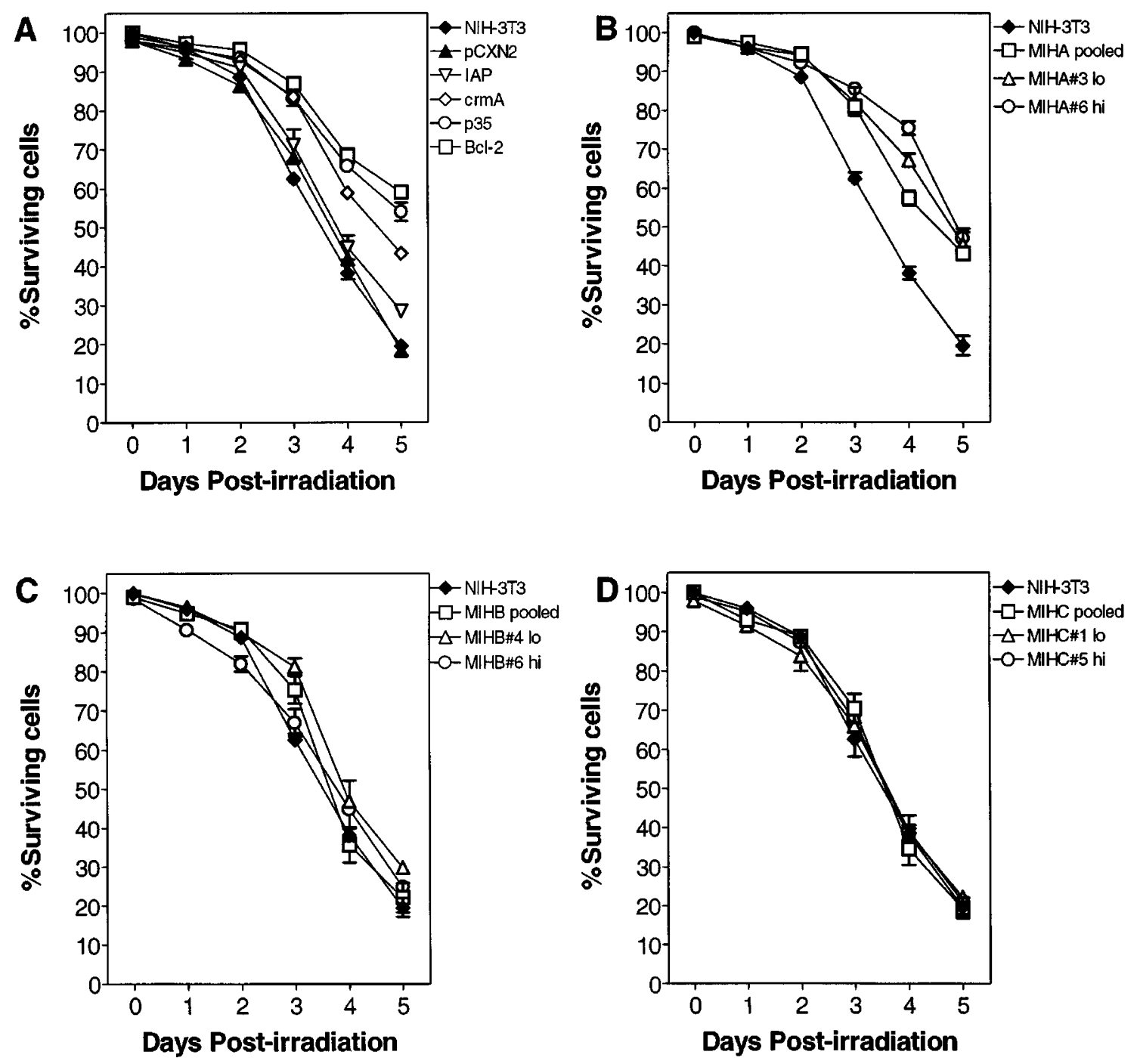

Figure $7 \gamma$-irradiation induced apoptosis in NIH3T3 cell stably expressing Bcl-2, CrmA, P35 and IAP (A), MIHA (B), MIHB (C) and MIHC (D). Data (means \pm S.E.M.) represent the percentage surviving cells as assessed by trypan blue exclusion. 'Pooled' denotes pooled populations of transfected cells; 'lo' denotes clones of cells with low levels of protein expression; 'hi' denotes clones of cells with higher levels of protein expression and clone numbers corresponding to those in Figure 3 are indicated. For Bcl-2, CrmA, P35 and IAP, only pooled population of transfected cells were used

Table 1 A summary of the relative efficiencies of $\mathrm{CrmA}$, P35 IAP, Bcl-2, MIH-A, MIH-B and MIH-C in inhibiting apoptosis in NIH3T3 cells following various death stimuli

\begin{tabular}{lc}
\hline Apoptotic stimuli & Relative inhibition \\
\hline ICE overexpression & $\mathrm{CrmA}>\mathrm{P} 35>\mathrm{IAP} \approx \mathrm{MIHA} \approx \mathrm{Bcl}-2>\mathrm{MIHB}>\mathrm{MIHC}$ \\
Nedd2 overexpression & $\mathrm{P} 35>\mathrm{Bcl}-2>\mathrm{IAP} \approx \mathrm{MIHB} \approx \mathrm{MIHC}>\mathrm{MIHA} \approx \mathrm{CrmA}^{1}$ \\
CPP32 overexpression & $\mathrm{P} 35>\mathrm{MIHA} \approx \mathrm{Bcl}-2 \approx \mathrm{IAP}>\mathrm{CrmA}^{1} \approx \mathrm{MIHC}^{1} \approx \mathrm{MIHB}^{1}$ \\
Serum withdrawal & $\mathrm{Bcl}-2>>\mathrm{P} 35>\mathrm{MIHA}>\mathrm{CrmA} \approx \mathrm{MIHB}^{1} \mathrm{MIHC}^{1}>\mathrm{IAP}^{1}$ \\
$\gamma$-irradiation & $\mathrm{Bcl}-2 \approx \mathrm{MIHA} \approx \mathrm{P} 35>\mathrm{CrmA}>\mathrm{IAP} \mathrm{M}^{1} \approx \mathrm{MIHB}^{1} \approx \mathrm{MIHC}^{1}$ \\
\hline
\end{tabular}

${ }^{1}$ No significant inhibition of apoptosis was observed

and assessed for viability. Detached cells in the culture supernatants were collected by centrifugation. Adherent cells were washed with PBS and trypsinised. Fractions from supernatant, washes and trypsinised cells were pooled and recovered by centrifugation. Pellets were resuspended in $0.2-0.5 \mathrm{ml}$ PBS and viability and apoptosis determined by trypan blue exclusion and nuclear staining respectively. For $\gamma$-irradiation, NIH3T3 cells were plated at a density of $6 \times 10^{5}$ cells per D60 dish. Semi-confluent cell monolayers were washed twice in PBS and refed in fresh DMEM. Cells were irradiated with 30 Gy of $\gamma$ irradiation from a ${ }^{137} \mathrm{Cs}$, harvested every $24 \mathrm{~h}$ for 5 days and the 
percentage cell survival estimated as above. Harvested cells were also assessed for apoptotic morphology by staining with 4,6diamidino-2-phenylindole (DAPI). A drop of the cell suspension was mixed with a drop of $50 \%$ formaldehyde $/ 50 \%$ DAPI mix and mounted with a coverslip. Slides were viewed under a fluorescence microscope and the percentage of apoptotic cells counted.

\section{Immunoblotting}

Proteins were electrophoresed on $10 \%$ polyacrylamide/SDS gels and transferred to nitrocellulose membrane using a semi-dry blotting apparatus. Membranes were blocked overnight at $4{ }^{\circ} \mathrm{C}$ in $5 \%$ skim milk/ PBS-T ( $1 \times$ PBS, $0.05 \%$ Tween 20$)$ and then probed with an anti-FLAG antibody (Kodak) diluted 1:1000 followed by a 1:2000 dilution of a anti-mouse IgG, conjugated with horseradish peroxidase (Amersham). After appropriate washing steps, signals were detected using ECL (Amersham). Activation of caspases was analyzed by immunoblot analysis of transiently transfected NIH3T3 and N18 cells, $18 \mathrm{~h}$ following lipofection. The conditions for the immunoblotting of caspases are described elsewhere (Harvey et al, 1997).

\section{RNA isolation and expression analysis}

Total RNA was prepared from cultured cells and mouse tissues using RNAzolB according to the instructions provided by the manufacturers (Tel-Test, Inc.). Samples were electrophoresed on $1.2 \%$ agarose/ 2.2 $\mathrm{M}$ formaldehyde gels, transferred to Biodyne A membrane (Pall) and hybridised to probes labelled with $\alpha^{32} \mathrm{P}$-dCTP by random priming.

\section{Acknowledgements}

We are grateful to David Vaux, David Pickup, Lois Miller, Douglas Miller, Vishva Dixit and Junichi Miyazaki for either providing various plasmid constructs or permitting the use of plasmids originating in their laboratories. We also thank Anthony Uren, Christine Hawkins and David Vaux for many helpful discussions, and Gayathri Parasivam for excellent technical assistance. This work was supported by a project grant from the National Health and Medical Research Council of Australia (no. 960532), and a Wellcome Trust Senior Research Fellowship in Medical Science to S.K.

\section{References}

Allet B, Hochmann A, Martinou I, Berger A, Missotten M, Antonsson B, Sadoul R, Martinou J-C and Bernasconi L (1996) Dissecting processing and apoptotic activity of a cysteine protease by mutant analysis. J. Cell Biol. 135: 479-486

Alnemri ES, Livingston DJ, Nicholson DW, Salvesen G, Thornberry NA, Wong WW and Yuan J (1996) Human ICE/CED-3 protease nomenclature. Cell 87: 171

Beidler DR, Tewari M, Friesen PD, Poirier G and Dixit VM (1995) The baculovirus p35 protein inhibits Fas- and tumor necrosis factor-induced apoptosis. J. Biol. Chem. 270: $16526-16528$

Birnbaum MJ, Clem RJ and Miller LK (1994) An apoptosis-inhibiting gene from a nuclear polyhedrosis virus encoding a peptide with cys/his sequence motifs. J. Virol. 68: 2521-2528

Bump NJ, Hackett M, Hugunin M, Seshagiri S, Brady K, Chen P, Ferenz C, Franklin S, Ghayur T, Li P, Licari P, Mankovich J, Shi L, Greenberg AH, Miller LK and Wong WW (1995) Inhibition of ICE family of proteases by baculovirus antiapoptotic protein p35. Science 269: 1885-1888

Clem RJ, Hardwick JM and Miller LK (1996) Anti-apoptotic genes of baculoviruses. Cell Death Differ. 3: 9-16

Clem RJ and Miller LK (1994) Control of programmed cell death by baculovirus genes p35 and IAP. Mol. Cell. Biol. 14: 5212-5222

Cory S (1995) Regulation of lymphocyte survival by the Bcl-2 gene family. Annu. Rev. Immunol. 13: 513-543
Crook NE, Clem RJ and Miller LK (1993) An apoptosis-inhibiting baculovirus gene with a zinc finger-like motif. J. Virol. 67: 2168-2174

Duckett CS, Nava VE, Gedrich RW, Clem RJ, Van Dongen JL, Gilfillan MC, Shiels H, Hardwick JM and Thomspon CB (1996) A conserved family of cellular genes related to the baculovirus iap gene and encoding apoptosis inhibitors. EMBO J. 15: $2685-2694$

Fernandes-Alnemri T, Litwack G and Alnemri ES (1994) CPP32, a novel human apoptotic protein with homology to Caenorhabditis elegans cell death protein Ced-3 and mammalian interleukin-1 $\beta$-converting enzyme. J. Biol. Chem. 269: $30761-30764$

Gagliardini V, Fernandez P-A, Lee RKK, Drexler HCA, Rotello RJ, Fishman MC and Yuan J (1994) Prevention of vertebrate neuronal death by crmA gene. Science 263: $826-828$

Harvey NL, Butt A and Kumar S (1997) Functional activation of Nedd2/lCH-1 (caspase-2) is an early process in apoptosis. J. Biol. Chem. 272: 13134-13139

Hawkins CJ, Uren AG, Häcker G, Medcalf RL and Vaux DL (1996) Inhibition of interleukin $1 \beta$-converting enzyme-mediated apoptosis of mammalian cells by baculovirus IAP. Proc. Natl. Acad. Sci. USA 93: 13786-13790

Hay BA, Wassarman DA and Rubin GM (1995) Drosophila homologs of baculovirus inhibitor of apoptosis proteins function to block cell death. Cell 83: 1253-1262

Hay BA, Wolff T and Rubin GM (1994) Expression of baculovirus p35 prevents cell death in Drosophila. Development 120: 2121-2129

Kerr JFR, Wyllie AH and Currie AR (1972) Apoptosis: a basic biological phenomenon with wide-ranging implications in tissue kinetics. Br. J. Cancer 26: 239-257

Kumar S (1995) ICE-like proteases in apoptosis. Trends Biochem. Sci. 20: 198-202

Kumar S, Kinoshita M, Noda M, Copeland NG and Jenkins NA (1994) Induction of apoptosis by the mouse Nedd2 gene, which encodes a protein similar to the product of the Caenorhabditis elegans cell death gene ced-3and the mammalian IL-1 $\beta$-converting enzyme. Genes Develop. 8: 1613-1626

Kumar S and Lavin MF (1996) The ICE family of cysteine proteases as effectors of cell death. Cell Death Differ. 3: 255-267

Liston P, Roy N, Tamai K, Lefebvre C, Baird S, Cherton-Horvat G, Farahani R, McLean M, Ikeda J, Mackenzie A and Korneluc RG (1996) Suppression of apoptosis in mammalian cells by NAIP and a related family of IAP genes. Nature 379: $349-353$

Martin SJ and Green DR (1995) Protease activation during apoptosis: Death by a thousand cuts. Cell 82: $349-352$

Martinou I, Fernandez P-A, Missotten M, White E, Allet B, Sadoul R and Martinou J-C (1995) Viral proteins E1B19K and p35 protect sympathetic neurons from cell death induced by NGF deprivation. J. Cell Biol. 128: 201-208

Miura M, Zhu H, Rotello R, Hartwieg EA and Yuan J (1993) Induction of apoptosis in fibroblasts by IL- $1 \beta$-converting enzyme, a mammalian homolog of the $C$. elegans cell death gene ced-3. Cell 75: 653-660

Niwa H, Yamamura K and Miyazaki J (1991) Efficient selection of high-expression transfectants with a novel eukaryotic vector. Gene 108: 193-200

Quan LT, Caputo A, Bleackley RC, Pickup DJ and Salvesen GS (1995) Granzyme B is inhibited by the cowpox virus serpin cytokine response modifier A. J. Biol. Chem. 270: $10377-10379$

Rabizadeh S, LaCount DJ, Friesen PD and Bredesen DE (1993) Expression of the baculovirus p35 gene inhibits mammalian neural cell death. J. Neurochem. 61 : $2318-2321$

Ray CA, Black RA, Kronheim SR, Greenstreet TA, Sleath PR, Salvesen GS and Pickup DJ (1992) Viral inhibition of inflammation: Cowpox virus encodes an inhibitor of the interleukin-1 $\beta$ converting enzyme. Cell 69: 597-604

Rothe M, Pan M-G, Henzel WJ, Ayres TM and Goeddel DV (1995) The TNFR2-TRAF signalling complex contains two novel proteins related to baculoviral inhibitor of apoptosis proteins. Cell 83: 1243-1252

Roy N, Mahadevan MS, McLean M, Shutler G, Yaraghi Z, Farahani R, Baird S, Besner-Johnson A, Lefebvre C, Kang X, Salih M, Aubry H, Tamai K, Guan X, Ioannou P, Crawford TO, de Jong PJ, Surh L, Ikeda J-E, Korneluk RG and MacKenzie A (1995) The gene for neuronal apoptosis inhibitory protein is partially deleted in individuals with spinal muscular atrophy. Cell 80: 167-178

Shen Y and Shenk TE (1995) Viruses and apoptosis. Curr. Opi. Genet. Develop. 5: 105-111

Shu H-B, Takeuchi M and Goeddel DV (1996) The tumor necrosis factor receptor 2 signal transducers TRAF2 and C-IAP1 are components of the tumor necrosis factor receptor 1 signalling complex. Proc. Natl. Acad. Sci. USA 93: 1397313978 
Sugimoto A, Friesen PD and Rothman JH (1994) Baculovirus p35 prevents developmentally programmed cell death and rescues a ced-9 mutant in the nematode Caenorhabditis elegans. EMBO J. 13: 2023-2028

Tewari M and Dixit VM (1995a) Fas- and Tumour Necrosis Factor-induced apoptosis is inhibited by the poxvirus crmA gene product. J. Biol. Chem. 270: 3255-3260

Tewari M, Telford WG, Miller RA and Dixit VM (1995b) CrmA, a poxvirus-encoded serpin, inhibits cytotoxic T-lymphocyte-mediated apoptosis. J. Biol. Chem. 270: 22705-22708

Uren AG, Pakusch M, Hawkins CJ, Puls KL and Vaux DL (1996) Cloning and expression of apoptosis inhibitory protein homologs that function to inhibit apoptosis and/or bind TRAFs. Proc. Natl. Acad. Sci. USA 93: 4976-4978
Vaux DL and Strasser A (1996) The molecular biology of apoptosis. Proc. Natl. Acad. Sci. USA 93: 2239-2244

Wang L, Miura M, Bergeron L, Zhu H and Yuan J (1994) Ich-1, and /ce/ced-3-related gene, encodes both positive and negative regulators of programmed cell death. Cell 78: $739-750$

Xue D and Horvitz HR (1995) Inhibition of the Caenorhabditis elegans cell death protease CED-3 by a CED-3 cleavage site in baculovirus p35 protein. Nature 377: $248-251$ 